\title{
Three-Dimensional Confinement in the Conduction Band Structure of InP
}

\author{
C. S. Menoni, L. Miao, and D. Patel \\ Department of Electrical and Computer Engineering, Colorado State University, Fort Collins, Colorado 80523-1373
}

O. I. Mic'ic' and A. J. Nozik

National Renewable Energy Laboratory, Golden, Colorado 80401-3393

(Received 30 November 1999)

\begin{abstract}
Strong quantum confinement in InP is observed to significantly reduce the separation between the direct and indirect conduction band states. The effects of three-dimensional confinement are investigated by tailoring the initial separation between conduction band states using quantum dots (QDs) of different sizes and hydrostatic pressure. Analyses of the QD emission spectra show that the $X_{1 c}$ states are lowest in energy at pressures of $\sim 6 \mathrm{GPa}$, much lower than in the bulk. The transition to the $X_{1 c}$ states can be explained by either a sequence of $\Gamma-L$ and $L-X$ crossings, or by the crossover between strongly coupled $\Gamma$ and $X$ states.
\end{abstract}

PACS numbers: 71.24.+q, 71.55.Eq, 73.20.Dx, 78.55.Cr

Quantum confinement has dramatic effects on the optical and electronic properties of semiconductors. Quantum confinement gives rise to electron states whose energy can be varied with the confinement dimensions, and dramatically changes the density of states from steplike in one-dimensionally confined quantum well systems to atomiclike in three-dimensionally confined quantum dots (QDs). The changes in the density of states and the possibility of tailoring the band gap have attracted attention for the application of quantum confined systems in the design of optoelectronic devices.

In zinc blende quantum well structures, quantum confinement has also been found to alter the electronic structure. Increased carrier confinement leads to (i) an electronic configuration in which multiple quantum wells (MQWs) of direct band-gap semiconductors become intrinsically indirect, i.e., $L_{1 c}$ or $X_{1 c}$ are the lowest conduction band states in the well, or (ii) a type II conduction band alignment with the lowest indirect conduction band state located in the barrier and the highest valence band state in the well. The former case is obtained in InGaP/InAlP MQWs and the latter is observed in GaAs/AlAs MQWs for well widths less than $35 \AA[1,2]$.

In three-dimensionally confined systems, model calculations predict quantum confinement to significantly alter the electronic structure of zinc blende semiconductors as the lower effective mass $\Gamma_{1 c}$ state rises to higher energy much faster than the higher effective mass $L_{1 c}$ and $X_{1 c}$ states $[3,4]$. In strained III-V QDs grown epitaxially, the interplay between carrier confinement and strain could result in either a type II electronic configuration with $X_{1 c}$-derived states in the capping layer and holes in $\Gamma_{1 v}$-derived QD states, or in a type I configuration where the indirect states in the QD are lowest in energy [4]. Instead, in freestanding colloidal QDs, only an intrinsically indirect electronic configuration is possible [3,5]. The model calculations predict $\Gamma-X$ transitions to be observed in freestanding GaAs QDs with diameter $<40 \AA$ [6], and in InP QDs at pressures around $7 \mathrm{GPa}$, as confinement alone is not sufficient to lower the $X$-like states below $\Gamma$-like states even for zero dimension [3].

In this Letter, we present the first experimental evidence showing that strong quantum confinement significantly reduces the separation between direct and indirect conduction band states in InP. To determine the effects of three-dimensional confinement, we used QDs of different size to tailor the initial separation among $\Gamma_{1 c^{-}}, L_{1 c^{-}}$, and $X_{1 c}$-like states and, in addition, applied hydrostatic pressure to further modify the electronic structure and reveal the indirect states at high pressures. Since in bulk III-V materials $\Gamma_{1 c}, L_{1 c}$, and $X_{1 c}$ shift at characteristics rates of $\sim 80 \mathrm{meV} / \mathrm{GPa}, \sim 50 \mathrm{meV} / \mathrm{GPa}$, and $\sim-10 \mathrm{meV} / \mathrm{GPa}$, respectively, the pressure rate of change of the emission peak $\left(d E_{p} / d p\right)$ has been used as a signature for identifying the direct and indirect states [7].

The colloidally grown InP QDs were synthesized from a mixture of indium trichloride, tris-(trimethylsilyl)phosphine $\left[\mathrm{P}\left(\mathrm{SiMe}_{3}\right)_{3}\right]$, trioctylphosphine oxide (TOPO), and trioctylphosphine (TOP) at room temperature and then heated at high temperature $[8,9]$. The resulting InP QD samples consisted of InP nanocrystals that crystallize in the zinc blende structure, and whose surface was capped by TOPO $[8,9]$. The QDs were further etched in a methanolic solution of HF that passivates the surface, enhancing the InP band edge emission and reducing the sub-band-gap emission associated with surface states [8]. Selective precipitation of the QD solution was used to obtain three different samples with mean QD diameters $\langle d\rangle$ of 32, 35, and $56 \AA$, respectively, and standard deviation $\left\langle\sigma_{d}\right\rangle=10 \%$.

For the high pressure experiments, the InP QDs were spun over a thin $(<50 \mu \mathrm{m})$ piece of InP bulk (Zn-doped, $\left.p=5 \times 10^{17} \mathrm{~cm}^{-3}\right)$ and loaded into the pressure chamber of a diamond anvil cell. A second InP bulk sample also loaded in the chamber was used to track the emission of bulk $\mathrm{InP}$ at different pressures and to perform a background subtraction which considerably improved the 
QD signal to noise ratio at high pressures, where it became very weak. The two samples, and a ruby chip used to monitor the pressure, were surrounded by solid argon which served as the pressure transmitting medium and allowed hydrostatic conditions to reach pressures in excess of $7 \mathrm{GPa}$. The changes in the electronic structure of the QDs and bulk InP were monitored from the shift of the photoluminescence (PL) emission excited by the $410 \mathrm{~nm}$ output from a frequency doubled Ti:sapphire laser. The emission was collected and analyzed using a spectrometer and liquid nitrogen cooled charge-coupled device detector.

Typical emission spectra for the $32 \AA$ QDs at different pressures is shown in Fig. 1(a). At atmospheric pressure, the spectra is composed of a PL peak at $1.95 \mathrm{eV}$ which corresponds to the band-to-band (BB) emission and a second emission peak at $1.7 \mathrm{eV}$ which originates from surface states (SS) associated with surface defects $[5,8,10,11]$. Fitting Gaussian functions to the spectra allowed the determination of the peak energy, integrated intensity, and full width at half maximum (FWHM) of each emission band. The BB-PL emission is broad with a FWHM $\sim 150 \mathrm{meV}$, reflecting the size distribution of the colloidally grown InP QDs. The BB-PL peak energy blueshifts with pressure initially at a rate $d E_{p} / d p \sim 80 \mathrm{meV} / \mathrm{GPa}$, slightly larger than that measured in bulk InP $\left(d E_{p} / d p=65 \mathrm{meV} / \mathrm{GPa}\right)$. At higher pressures, $d E_{p} / d p$ decreases and becomes slightly negative above $6 \mathrm{GPa}$, as shown in Fig. 2(a). Above $7 \mathrm{GPa}$, the QD BB emission is basically quenched. The BB-PL peak behavior is completely reversible as shown by the open symbols in Fig. 2(a). The changes in $d E_{p} / d p$ are also accompanied by changes in the PL intensity and FWHM, shown in Fig. 2(b). The PL intensity is almost constant to $\sim 2 \mathrm{GPa}$ where it starts to decrease and reaches a value $\sim 2$ orders of magnitude smaller than its atmospheric pressure value at the highest pressure. The FWHM increases by $50 \%$ over the whole pressure range; however, its change is more prominent above $4 \mathrm{GPa}$. The SS PL, $300 \mathrm{meV}$ below the BB emission, also blueshifts but at a lower rate; $d E_{p} / d p$ also decreases with pressure,
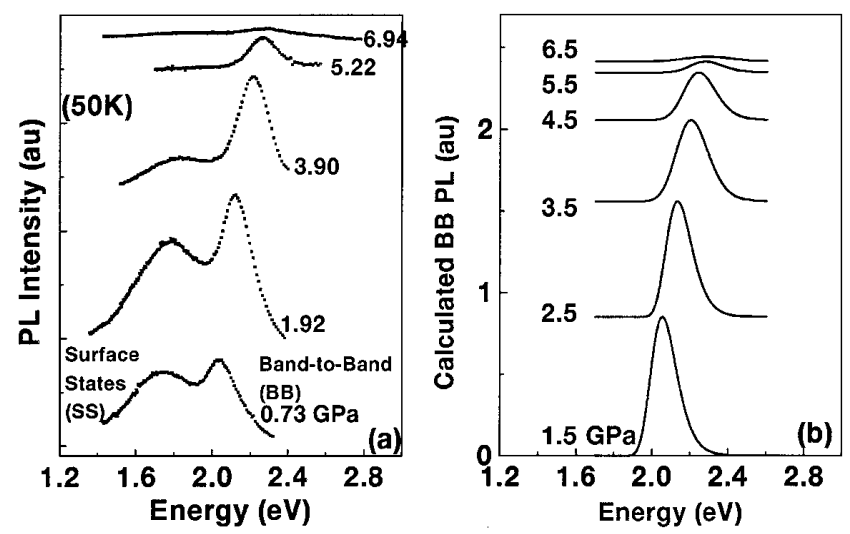

FIG. 1. (a) Emission spectra of $32 \AA$ InP QDs at different pressures. (b) Simulated BB emission obtained when a sequence of $\Gamma-L-X$ transitions occur at high pressure. undertracking the behavior of the BB emission. The lower value of $d E_{p} / d p$ in the SS emission compared to the $\mathrm{BB}$ emission is not surprising as these are deep states which are generally less sensitive to pressure. The SS also disappears when the main BB emission quenches at $\sim 7 \mathrm{GPa}$.

The BB-PL behavior just described for the $32 \AA \mathrm{QD}$ sample is also observed in the 35 and $56 \AA$ QDs, as shown in Fig. 3. The most obvious difference in the pressure behavior of the QDs compared to bulk InP is the bowing of the BB-PL peak energy with pressure which contrasts with the linear behavior of the bulk InP over the same pressure range. The bowing in the QD data arises from changes in $d E_{p} / d p$ which reduces from $\sim 80$ to $\sim 40 \mathrm{meV} / \mathrm{GPa}$ at $\sim 3 \mathrm{GPa}$ and becomes constant or slightly negative with pressure at $\sim 6 \mathrm{GPa}$ in the 32 and $35 \AA$ samples. In the $56 \AA$ QD sample, $d E_{p} / d p$ changes from 75 to $40 \mathrm{meV} / \mathrm{GPa}$ at $4 \mathrm{GPa}$. The second decrease in $d E_{p} / d p$ is not observed as the emission is quenched at lower pressures than in the smaller QD samples. At the onset of the first change in $d E_{p} / d p$, the BB-PL intensity starts to decrease, and markedly reduces at $\sim 6 \mathrm{GPa}$ when the second change in $d E_{p} / d p$ occurs. The decrease in $d E_{p} / d p$ and BB-PL intensity with pressure are signatures
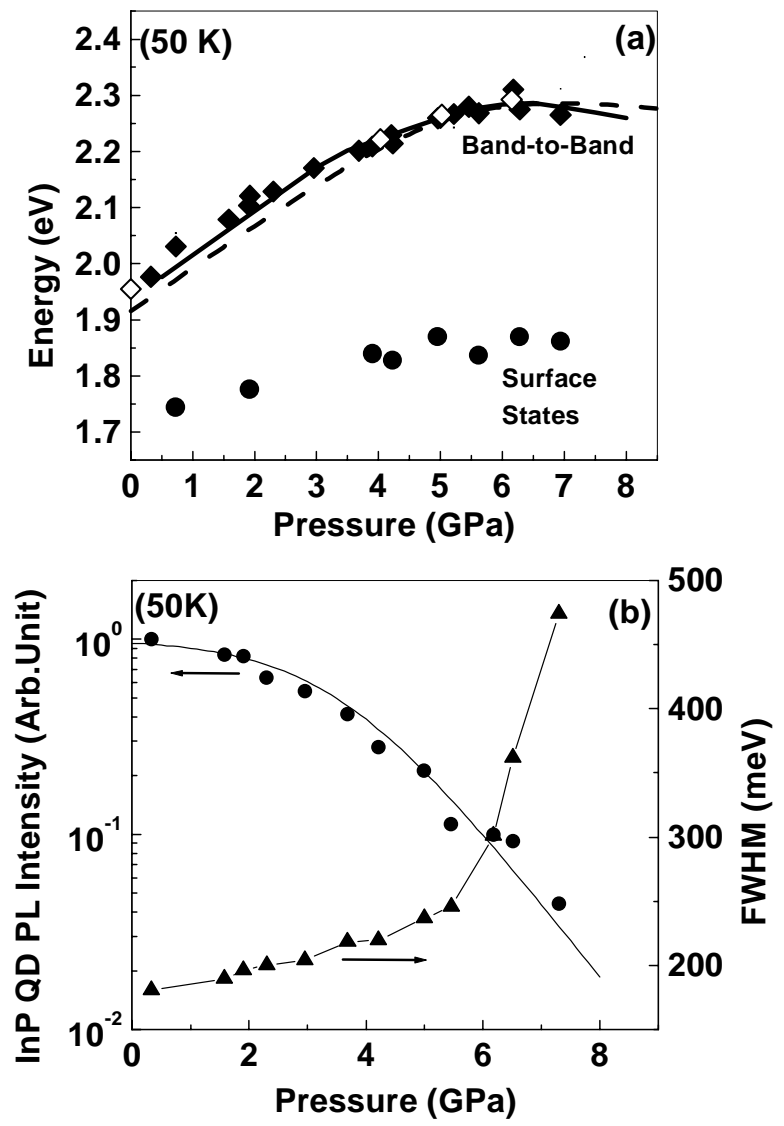

FIG. 2. (a) Variation of the BB-PL and SS-PL peak energy for $32 \AA$ InP QDs; solid symbols $(\diamond)$ : upstroke; open symbols $(\diamond)$ : downstroke. The dotted and solid lines are the results of a fit with a simulation that considers $\Gamma-X$ transfer and $\Gamma-L-X$ transfer, respectively. (b) Pressure dependence of the integrated BB-PL emission $(\mathbf{O})$ and FWHM $(\boldsymbol{\Delta})$. 
of carrier transfer to indirect conduction band states. The initial change in $d E_{p} / d p$ could be interpreted as being due to a $\Gamma-L$ transition, and the second change could result from an $L-X$ transition. The significant reduction in the PL intensity at $\sim 6 \mathrm{GPa}$ is also a strong indication of carrier transfer to the lower oscillator strength indirect $X_{1 c}$ states. But the band crossing behavior and associated intensity changes of InP QDs may be more complex than just described, depending on the strength of $\Gamma-L-X$ intervalley mixing. In the presence of intervalley mixing, the conduction band states near a transition show an anticrossing behavior which is reflected in hydrostatic pressure experiments as a splitting of optical transitions and smearing out of their pressure dependence $[12,13]$. De- pending on the strength of the mixing, the QD peak emission energy shift with pressure could significantly depart from the bulk behavior. The overall intensity profile of the ensemble QD PL emission should also be considerably modified. $\Gamma-L-X$ mixing, coupled with the considerable reduction in the emission efficiency of $X_{1 c}$-like states, may prevent the observation of a pure $X$-like behavior in InP QDs.

To visualize the behavior of the QD PL peak energy with pressure, we simulated the QD emission and tracked the position of its peak at different pressures. The ensemble PL emission intensity was obtained by superimposing the single-dot emission of a collection of QDs of diameter $d$, average diameter $\langle d\rangle$, and standard deviation $\sigma_{d}$, which follows a Gaussian distribution in dot size [10]:

$$
\overline{I_{\mathrm{PL}}}\left(E, E_{\mathrm{exc}},\langle d\rangle, p\right)=\sum_{d>d_{c}\left(E_{\mathrm{exc}}\right)} \alpha\left(E_{\mathrm{exc}}, d\right)\left[\frac{A(p)}{\sqrt{2 \pi} \sigma_{\mathrm{PL}}} e^{\left\{E-\left[E_{g}(d, p)-\Delta(d)\right]^{2}\right\} /\left(2 \sigma_{\mathrm{PL}}^{2}\right)}\right]\left[\frac{1}{\sqrt{2 \pi} \sigma_{d}} e^{(d-\langle d\rangle)^{2} /\left(2 \sigma_{d}^{2}\right)}\right] .
$$

In the single-dot emission, $E_{g}(d, p)$ is the excitonic band-gap energy, $\sigma_{\mathrm{PL}}$ is the intrinsic broadening, and $\Delta(d)$ is the single-dot redshift which varies with $d$ as $\Delta(d)=9.5 / d^{1.96}$, where $d$ is expressed in units of $\AA$ [10]. $\alpha\left(E_{\text {exc }}, d\right)$ is the single-dot absorption coefficient at the excitation energy $E_{\text {exc }}$, and depends on dot diameter as $\alpha \sim\left(a_{B} / d\right)^{3}$, where $a_{B}$ is the Bohr radius. The sum over $d$ in Eq. (1) is limited to those values satisfying the condition $E_{g}(d)<E_{\mathrm{exc}}$, which is our case at all pressures for all three samples. $A(p)$ is the amplitude which was

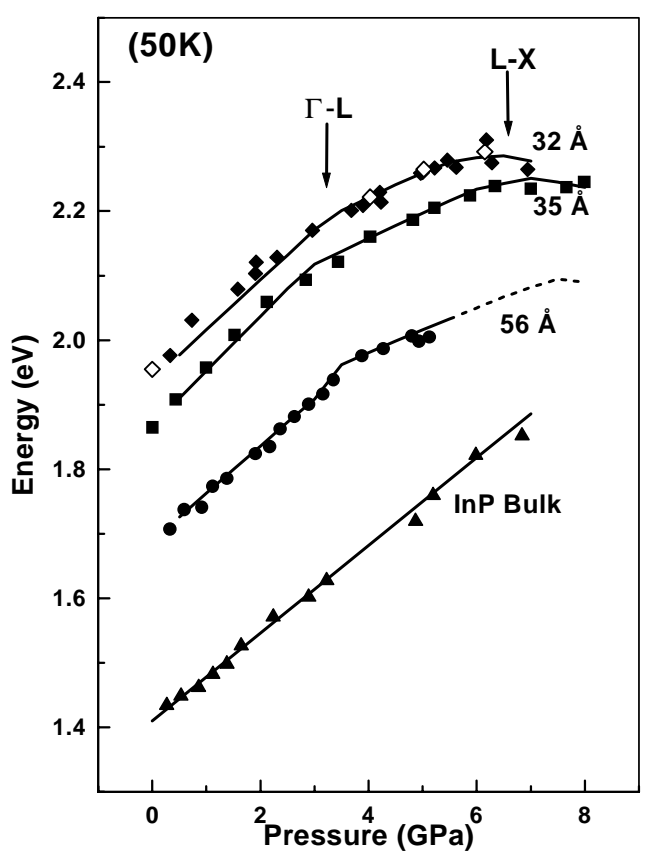

FIG. 3. Variation of the BB-PL peak with pressure for $32 \AA$ $(\bullet), 35 \AA(\boldsymbol{\square})$, and $56 \AA(\bigcirc) \operatorname{InP}$ QDs and InP bulk $(\mathbf{\Delta})$. The arrows indicate the pressures at which $d E_{p} / d p$ changes in the QDs. The solid lines are obtained from the fit of the data with the $\Gamma-L-X$ simulation. adjusted at atmospheric pressure to match the measured $\mathrm{BB}$ emission. The dependence of the $\Gamma_{1 c}$-like $E_{g}$ on dot size was taken equal to $1.42+15.10 / d^{0.93}$ [5].

To model the pressure behavior of the ensemble PL, we first considered that only a $\Gamma-X$ transition takes place. The pressure dependence was incorporated in the single-dot excitonic band gap $E_{g}$, which varies with pressure as

$$
\begin{aligned}
E_{g}(p)= & E^{-}=E\left(P_{c}\right)+\frac{1}{2}\left(\alpha_{\Gamma}+\alpha_{X}\right)\left(p-P_{c}\right) \\
& -\left\{\left[\frac{1}{2}\left(\alpha_{\Gamma}-\alpha_{X}\right)\left(p-P_{c}\right)\right]^{2}+V_{\Gamma X}^{2}\right\}^{1 / 2} .
\end{aligned}
$$

$E_{g}(p)$ given in Eq. (2) is obtained from first order perturbation theory when two states, $\Gamma$ and $X$ in this case, interact or become mixed near a critical pressure $P_{c}$ [12]. $\alpha_{\Gamma}$ and $\alpha_{X}$ in Eq. (2) are the pressure coefficients of the unperturbed $\Gamma_{1 c}$ and $X_{1 c}$ states, away from $P_{c} . E\left(P_{c}\right)$ is the energy at which the unperturbed states would cross; i.e., $E_{\Gamma}\left(P_{c}\right)=E_{X}\left(P_{c}\right)$ and $V_{\Gamma X}$ is the interaction or mixing potential. In our simulation, $\alpha_{\Gamma}$ was elected to be the value of $d E_{p} / d p$ measured close to atmospheric pressure, and $\alpha_{X}$ was set equal to $-16 \mathrm{meV} / \mathrm{GPa}$, a typical value for the $X$ pressure coefficient, and equal to that measured in InAs/GaAs QDs $[1,12,14]$. Using $\alpha_{\Gamma}, \alpha_{X}$, and considering the $X$-excitonic band-gap energy to vary with dot size as $E_{X}^{\text {dot }}=2.3+135.8992 / d^{1.7165}$ [15], $P_{c}$ was calculated for each dot size within the ensemble. For QDs of $\langle d\rangle=32$ and $35 \AA, P_{c}$ equals 6.2 and $6.5 \mathrm{GPa}$, respectively [3], very close to the experimental value at which $d E_{p} / d p$ is found to be pressure independent. Since $P_{c}$ is size dependent, near the $\Gamma-X$ crossover the PL emission is composed of the emission from larger single dots whose conduction ground state is $\Gamma_{1 c}$-like and smaller ones in which $X_{1 c}$-like states are the lowest. To account for the superposition of markedly different emission states, we set in our simulation the peak amplitude of the $X_{1 c}$-like states 
to be 2 orders of magnitude smaller than that of $\Gamma_{1 c}$-like states, following the trend observed in the experiments. In the pressure dependence of Eq. (1), we also considered the effect of state mixing in the excitonic binding energy, which was calculated semiempirically from the binding energy of the $\Gamma$-like states taken to be equal to $\Delta(d)$ [16]. Leaving $V_{\Gamma X}$ as an undetermined parameter, we calculated the emission spectrum at different pressures [Fig. 1(b)], and fitted its peak energy variation with pressure to the experimental data for all three samples. The results of the least squares fit, shown by the dotted line in the $32 \AA$ data of Fig. 2(a), yield $V_{\Gamma X}=70 \mathrm{meV}$. This large value of $V_{\Gamma X}$ gives the band-gap dependence on pressure the sublinear behavior observed in the experiment. It also perturbs the $\Gamma_{1 c}$-like band gap close to atmospheric pressure, lowering $E_{g} \sim 0.04 \mathrm{eV}$ below the measured value, and predicts a pure $X_{1 c}$-like behavior to occur well beyond $7 \mathrm{GPa}$. However, it should be noted that, since $V_{\Gamma X}$ was obtained by setting $P_{c}$ for each dot in the ensemble at $\sim 6 \mathrm{GPa}$, its value represents an upper limit. This choice coupled with the uncertainty in $\alpha_{X}$ (which for the InP QDs cannot be directly measured as a pure $X$-like behavior is not observed) are likely to be responsible for the difference between the value of $V_{\Gamma X}$ determined from our analysis and that predicted by model calculations [3].

We also considered in our simulation the possibility of a $\Gamma-L$ crossing at lower pressures followed by an $L-X$ crossing at higher pressures. This scenario is possible in the QDs as the $L_{1 c}$-like states lie below $X_{1 c}$ at atmospheric pressure [5], provided the $\Gamma-L$ separation decreases faster than $\Gamma-X$. A $\Gamma-L-X$ sequence of electronic transitions would produce changes in $d E_{p} / d p$ similar to those observed experimentally. The $\Gamma-L$ transition was incorporated in the simulation by calculating $E_{g}$ in Eq. (2) assuming the unperturbed $\Gamma_{1 c}$ and $L_{1 c}$ states cross at a critical pressure $P_{c L}$ of $3.3,3.0$, and $3.6 \mathrm{GPa}$ equal to that where the first change in $d E_{p} / d p$ is observed in the 32,35 , and $56 \AA$ QDs, respectively. $\alpha_{L}$ was set equal to $d E_{p} / d p=40 \mathrm{meV} / \mathrm{GPa}$, the slope of the experimental data between 3 and $6 \mathrm{GPa}$. Selecting $P_{c L}$ and $\alpha_{L}$ for all three samples was equivalent to determining the variation of the excitonic band gap with QD diameter as was done in the $\Gamma-X$ simulation. $\alpha_{\Gamma}, \alpha_{X}$, and the $\Gamma-X$ separation at atmospheric pressure were kept the same as in the previous case; thus, Eq. (2) modeled the $L-X$ crossing around $6 \mathrm{GPa}$ where $d E_{p} / d p$ becomes independent of pressure. The solid lines in the data of Figs. 2(a) and 3 show the results of the fit when $\Gamma-L$ and $L-X$ transfer occur. Since the bowing in the emission peak behavior is mainly accounted for by the changes in $d E_{p} / d p$, the intervalley mixing potentials obtained from the fit $V_{\Gamma L}=2 \mathrm{meV}$ and $V_{L X}=10 \mathrm{meV}$ are smaller and comparable to those calculated in Ref. [3], and measured in InAs/GaAs QDs [12].

The above results undoubtedly show that a transition to $X_{1 c}$-like states occurs in InP QDs at $\sim 6 \mathrm{GPa}$, much lower than in bulk InP [17], reflecting the effects of quantum confinement on the electronic structure predicted by theory [3]. However, the detailed nature of this transition is not uniquely established, as a pure $\Gamma, L$, and $X$ pressure behavior is not distinct in the freestanding InP QDs. This is because, near a crossover, the ensemble emission is composed of the single-dot emission from larger $(d>\langle d\rangle)$ and smaller $(d<\langle d\rangle)$ QDs whose conduction ground states have markedly different pressure behavior. $\Gamma-L-X$ intervalley mixing even further modifies the collective pressure dependence of the emission. Accounting for these effects, the transition to $X_{1 c}$ states can be explained as a $\Gamma-L$ crossover followed by an $L-X$ crossover if the changes in $d E_{p} / d p$ are used as a signature of the $\Gamma_{1 c}, L_{1 c}$, and $X_{1 c}$ states, or by the crossing of strongly mixed $\Gamma_{1 c}$ and $X_{1 c}$ states. Nevertheless, in any case, the 2 orders of magnitude reduction in the integrated intensity at $\sim 6 \mathrm{GPa}$ is a strong indication of carrier transfer to the $X_{1 c}$ states which are lowest in energy at the highest pressures.

The authors would like to acknowledge Dr. H. Fu and Dr. A. Zunger for useful discussions. The work at CSU was supported by the National Science Foundation, and that at NREL by the U.S. Department of Energy, Office of Basic Energy Sciences, Chemical Sciences Division.

[1] D. Patel, K. Interholzinger, P. Thiagarajan, G. Y. Robinson, and C. S. Menoni, Phys. Rev. B 53, 12633 (1996).

[2] K. Meissner et al., Appl. Phys. Lett. 59, 259 (1991).

[3] H. Fu and A. Zunger, Phys. Rev. Lett. 80, 5397 (1998).

[4] A. Franceschetti and A. Zunger, Phys. Rev. B 52, 14664 (1995).

[5] H. Fu and A. Zunger, Phys. Rev. B 56, 1496 (1997).

[6] A. Franceschetti and A. Zunger, Appl. Phys. Lett. 68, 3455 (1996).

[7] I. L. Spain, Contemp. Phys. 28, 523 (1987).

[8] O. I. Micic, C. J. Curtis, K. M. Jones, J. R. Sprague, and A. J. Nozik, J. Phys. Chem. 98, 4966 (1994).

[9] O. I. Micic et al., J. Phys. Chem. 99, 7754 (1995); O. I. Micic, J. Sprague, Z. Lu, and A. J. Nozik, Appl. Phys. Lett. 68, 3150 (1996).

[10] O. I. Micic et al., J. Phys. Chem. 101, 4904 (1997).

[11] A stronger contribution of the SS emission in the $32 \AA$ sample is observed in these experiments because the sample was not pretreated with an HF solution immediately before loading.

[12] G. H. Li, A. R. Goñi, K. Syassen, O. Brandt, and K. Ploog, Phys. Rev. B 50, 18420 (1994).

[13] W. Shan et al., Phys. Rev. Lett. 82, 1221 (1999).

[14] D. J. Wolford and J. A. Bradley, Solid State Commun. 53, 1069 (1985).

[15] $\mathrm{H} . \mathrm{Fu}$ (private communication).

[16] The pressure dependent exciton binding energy was calculated as $\Delta_{\Gamma X}=\Delta(d)[\mu(p) / \mu(p=0)]$, where $\mu(p)$ is the exciton reduced effective mass. The functional dependence of $\mu(p)$ on $V_{\Gamma X}$ and $E_{g}(p)$ is the same as given in Eq. (8) of Ref. [12]. At $p=0, \mu_{\Gamma}=0.07 m_{0}$, and $\mu_{X}=0.144 m_{0}$ for the $\Gamma$ and $X$ states, respectively.

[17] S. Ernst, A. R. Goñi, K. Syassen, and M. Cardona, Phys. Rev. B 53, 1287 (1996). 